\title{
Inflation without a beginning: a null boundary proposal
}

\author{
Anthony Aguirre \\ School of Natural Sciences, Institute for Advanced Study Princeton, New Jersey 08540, USA* \\ Steven Gratton \\ Joseph Henry Laboratories, Princeton University, Princeton, New Jersey 08544, USA
}

(Dated: February 7, 2008)

\begin{abstract}
We develop our recent suggestion that inflation may be made past eternal, so that there is no initial cosmological singularity or "beginning of time". Inflation with multiple vacua generically approaches a steady-state statistical distribution of regions at these vacua, and our model follows directly from making this distribution hold at all times. We find that this corresponds (at the semi-classical level) to particularly simple cosmological boundary conditions on an infinite null surface near which the spacetime looks de Sitter. The model admits an interesting arrow of time that is well-defined and consistent for all physical observers that can communicate, even while the statistical description of the entire universe admits a symmetry that includes time-reversal. Our model suggests, but does not require, the identification of antipodal points on the manifold. The resulting "elliptic" de Sitter spacetime has interesting classical and quantum properties. The proposal may be generalized to other inflationary potentials, or to boundary conditions that give semi-eternal but non-singular cosmologies.

PACS numbers: 98.80.Cq, 04.20.Gz, 04.62.+v
\end{abstract}

\section{INTRODUCTION}

The ancient philosophical question of whether the universe is finite or infinite in time, and whether time "had a beginning", entered the domain of scientific study during the 20th century with the development of generally relativistic cosmologies in which the universe is homogeneous and expanding, as observed on the largest accessible scales.

Chief among these cosmologies, and representing opposite answers to the question of the universe's beginning, were the classical Big-Bang and Steady-State. As FRW models, both are based on some form of the "Cosmological Principle" (CP) that the large-scale statistical properties of the universe admit spatial translational and rotational symmetries. The models differ greatly, however, in their time evolution. In the Big-Bang, the properties of the universe evolve in a finite time from a dense, singular initial state. In contrast, the Steady-State universe is said to obey the "Perfect Cosmological Principle" (PCP) in that it admits, in additional to spatial translational and rotational symmetries, a time-translation symmetry. Since all times are equivalent, there can be no "beginning of time", and the universe is infinite in duration.

Unlike their philosophical predecessors, the Big-Bang and Steady-State model were observationally distinguishable, and astronomical evidence eventually turned nearly all cosmologists away from the Steady-State. Moreover, theorems proven within General Relativity showed that the classical singularity of the Big-Bang cosmology was

\footnotetext{
*Electronic address: aguirre@ias.edu

†Electronic address: sgratton@princeton.edu
}

robust and could not be avoided by relaxing simplifying assumptions such as that of homogeneity. Thus the idea of a temporally finite universe with a singular initial epoch came to dominate cosmology. ${ }^{1}$ Attention has since focused on how this primordial singularity (where some presently unknown theory of quantum gravity presumably applies) could give rise to a classical "initial" state that could evolve into the observed universe.

The required "initial" classical state, however, seemed rather special: the universe had to have been extremely flat, and statistically homogeneous (i.e. obey the CP) on scales larger than the horizon size. The theory of inflation was devised and widely accepted as a solution to this problem of a special initial state: given inflation, a flat, homogeneous universe (with the necessary Gaussian scale-invariant density fluctuations) is an attractor. That is, within some inflating region of fixed, finite physical size, the CP holds more and more precisely with time. What is perhaps more surprising and less widely appreciated, however, is that in generic inflation models the universe also comes to obey, with ever-greater precision, the Perfect Cosmological Principle. This occurs because inflation is generically "semi-eternal": rather than ending globally at some time, inflation always continues in some regions, and the universe globally approaches a quasi-steady-state distribution of inflating and thermalized regions, the statistical description of which becomes

\footnotetext{
1 There have been a number of proposals for avoiding a beginning of time, but generally these involve either continuing through the cosmological singularity by invoking quantum gravitational effects, or modifying GR at the classical level. Our approach aims to develop a non-singular cosmology without appeal to either possibility.
} 
asymptotically independent of time [1].

Since inflation generically approaches a steady-state, it seems physically reasonable to ask whether the universe can simply be in an inflationary steady-state, thus avoiding a cosmological singularity or "beginning of time". Indeed, the possibility of truly eternal inflation was raised soon after inflation's invention, but no satisfactory model was immediately devised [2], and in subsequent years several theorems were formulated proving the geodesic incompleteness of models globally satisfying conditions seeming necessary for eternal inflation 3, 4]. These theorems suggested that inflationary cosmologies necessarily contain singularities, but the exact nature of the implied singularities was obscure.

In a recent paper, we constructed a counter-example to these theorems by providing a model for geodesically complete truly eternal inflation [5]. There, we analyzed the classical Steady-State model in detail, then extended our analysis to inflation. Here, we develop the model from a different standpoint, focusing on an eternal inflation in a double-well inflaton potential, and on the corresponding cosmological boundary conditions. Section motivates and develops our model, and describes its general features. Various aspects of the model are developed in subsequent sections: Section III discusses the arrow of time in our model, and elucidates the failure of the singularity theorems to forbid our construction; Sec. [IV discusses the cosmological boundary conditions, which are specified on a null surface; Sec. $\nabla$ discusses the relation of our model to the "antipodally identified" or "elliptic" interpretation of de Sitter spacetime that it suggests, and briefly discusses quantum field theory in elliptic de Sitter; Sec. VI discusses generalizations and extensions of our model. We summarize and conclude in Sec. VIII

\section{THE PROPOSAL}

In this section we develop an eternally inflating cosmology based on a double-well inflaton potential $U(\phi)$ with minima at $\phi_{t}$ and $\phi_{f}$, where $U\left(\phi_{f}\right)>U\left(\phi_{t}\right) \geq 0$. This sort of potential is posited in "old" inflation [6] or "open" inflation [7, 8]. We will first review semi-eternal double-well inflation, then extend this to eternal inflation, then analyze and address the geodesic completeness of the model.

\section{A. Semi-eternal "double-well" inflation}

A semi-eternally inflating cosmology naturally arises from a generic double-well potential [9, 10]. Consider some large comoving region in which, at an initial time $t=t_{0}$, the energy density is dominated by the inflaton $\phi$, with $\phi \simeq \phi_{f}$ and $\partial_{\mu} \phi \simeq 0$. Assume the spacetime to locally resemble de Sitter ("dS") space, with (for convenience) nearly-flat spatial sections, i.e. with a metric 11] approximated by

$$
d s^{2}=-d t^{2}+e^{2 H t}\left(d x^{2}+d y^{2}+d z^{2}\right) .
$$

In this background, bubbles of true vacuum $\phi_{t}$ nucleate at a fixed rate $\lambda$ per unit physical 4 -volume that depends upon the potential $U(\phi)$ [12]. The interior of each bubble looks like an open FRW cosmology to observers inside it. For a suitably designed $U(\phi)$ (as in open inflation [7, 8] ), there can be a slow-roll inflation epoch inside the bubble so that the FRW regions are nearly flat and homogeneous, and have scale-invariant density perturbations. One such region could therefore in principle represent our observable cosmological surroundings.

At any time $t$, we can derive the distribution of bubbles and inflating region within our comoving volume, with the aim of showing that the distribution approaches a steady-state. To avoid complications resulting from bubble collisions and the ambiguities in connecting the time-slicings within and outside bubbles, we concentrate on the statistics describing the inflating region outside of the bubbles. This region is necessarily unaffected by the bubbles' presence because they expand at the speed of light: both its global and local properties depend only on its initial state at $t_{0}$. But we may describe three effects of the bubble encroachment upon it.

First, let us consider the inflating region left at time $t$ by bubbles forming since $t_{0}$. This region must consist entirely of points each of which does not have a nucleation event in its past light-cone (PLC) going back to $t_{0}$. Denoting the volume of this PLC by $Q\left(t, t_{0}\right)$, it can then be shown that such points comprise a volume fraction

$$
f_{\text {inf }}=\exp [-\lambda Q] \simeq \exp \left[\frac{-4 \pi \lambda\left(t-t_{0}\right)}{3 H^{3}}\right]
$$

for $\left(t-t_{0}\right) \gg H^{-1}[9,10]$. Although $f_{\text {inf }} \rightarrow 0$ for large $t-t_{0}$, the spacetime is said to be eternally inflating because for small $\lambda$ the physical inflating volume within our comoving region nonetheless increases exponentially with time:

$$
V_{\text {inf }} \propto f_{\text {inf }} \exp (3 H t) \sim \exp (D H t)
$$

for any fixed $t_{0}$, with $D \equiv 3-4 \pi \lambda / 3 H^{4}$.

Second, one can show $[9$ that at fixed $t$ the distribution of inflating regions about any inflating point is described by a fractal of dimension $D$ (that is, the inflating volume $\left.V_{\text {inf }} \propto r^{3} f_{\text {inf }}(r) \propto r^{D}\right)$ up to a scale of order $r_{B}\left(t, t_{0}\right)$, where

$$
r_{B}\left(t, t_{0}\right)=H^{-1}\left[e^{H\left(t-t_{0}\right)}-1\right]
$$

is the physical radius at $t$ of a bubble nucleated at $t_{0}$.

Third, we may calculate, for a given point in the inflating region at time, the number per unit time $N(r, t)$ of incoming bubbles of physical radius $r$. This is

$$
N(r, t)=\frac{4 \pi \lambda r^{2}}{(1+H r)^{4}}
$$




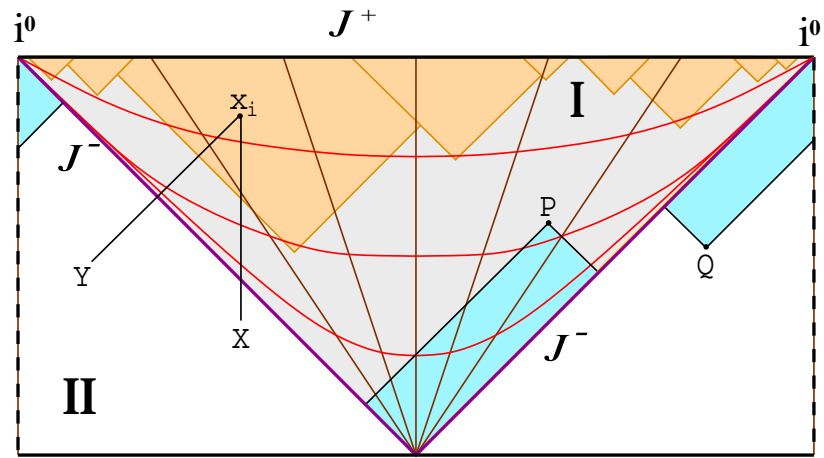

FIG. 1: Conformal diagram for de Sitter (dS) spacetime. Each point represents one point in $1+1 \mathrm{D} d \mathrm{~S}$, or half of a 2 -sphere in $3+1 \mathrm{D}$ dS. The left and right (dotted) edges are identified. The shaded region (region I) is covered by coordinates with flat spatial sections (spacelike lines) with spacelike infinity at $i_{0}$; the straight, timelike lines represent comoving geodesics. The null surface $\mathcal{J}^{-}$represents $t \rightarrow-\infty$. True-vacuum "test bubbles" (not disturbing the background spacetime) are darkly shaded and open toward future timelike infinity $\mathcal{J}^{+}$. Also shown are are the light-cones of points $\mathrm{P}$ and $\mathrm{Q}$ in regions I and II that open toward $\mathcal{J}^{-}$, and null ("Y") and timelike ("X") geodesic segments with an endpoint at $x_{i}$ and crossing $\mathcal{J}^{-}$.

for $r<r_{B}\left(t, t_{0}\right)$ and zero for $r>r_{B}$.

An observer within a bubble can never leave, but will eventually be encountered by an encroaching bubble wall after a typical time $\tau_{\text {coll }}$, where $\tau_{\text {coll }}^{-1}$ is related to the $r$ integral of Eq. (5) by some transformation between the bubble observer's proper time $\tau$ and cosmic time $t$. Since this rate depends on $t-t_{0}$, a patient and very sturdy observer could in principle discover the global time at which it formed by counting the frequency of incoming bubbles.

Now, as $t \rightarrow \infty$, four things occur. First, the nearlyflat spatial sections approach perfect flatness. Second, the incoming bubble rate $N(r, t)$ becomes homogeneous and independent of time on arbitrarily large physical scales. To see this, imagine that the rate is inhomogeneous at early times because bubble nucleation starts at different times in different regions. But since the impact rate depends on the initial time only for bubbles of radius greater than $r_{B}\left(t, t_{0}\right) \sim \exp \left[H\left(t-t_{0}\right)\right]$, it is then homogeneous and independent of time on arbitrarily large scales as $t \rightarrow \infty$. Third, and for essentially the same reason, the distribution of inflating region around any given inflating region also becomes homogeneous and independent of time on arbitrarily large scales. Fourth, observers within bubbles lose the information about the "global time" at which they exist (see Eq. (5)), and all bubbles become equivalent. Thus the physical description of the universe, relative to any fixed length scale such as $H^{-1}$, satisfies the Perfect Cosmological Principle arbitrarily well as $t \rightarrow \infty$.

\section{B. Eliminating the beginning}

The above semi-eternally inflating model can be made eternal by setting the "state" of the universe to be exactly that state approached by semi-eternal inflation: because the statistical properties depend only upon $t-t_{0}$, for specified conditions at $t_{0}$ the state at fixed $t$ with $t_{0} \rightarrow$ $-\infty$ is the same as that for $t \rightarrow \infty$ with fixed $t_{0}$.

The state so obtained has the four basic characteristics listed above: The spatial sections are exactly flat (outside of the bubbles), the bubble distribution (as characterized by the incoming bubble rate) is homogeneous and independent of time, as is the distribution of inflating regions about any inflating region, and the bubbles are all statistically identical. The inflating region is a fractal of dimension $D<3$ on all scales. This means that, although inflating regions exist, the global inflating fraction $f_{\text {inf }}$ is zero, just as the fraction of $3 \mathrm{D}$ Minkowski spacetime filled by an infinite 2-plane of finite thicknessan object of fractal dimension two - would vanish. (The zero probability that a randomly chosen point is in an inflating region accords with the fact [5] that within the PLC of each point there is an infinite 4-volume in which bubbles can nucleate toward that point.) Unlike the region filled by the plane, however, the inflating region is statistically homogeneous and isotropic, in that it exactly satisfies the "Conditional Cosmographic Principle" of Mandelbrot that the statistical description of the inflating region about any given inflating region is independent of the inflating region chosen (see Ref. [13] for a discussion of this and other aspects of "cosmological" fractals).

This model (essentially derived by Vilenkin [9]) would seem to have exactly the properties expected of an eternally inflating spacetime, has been straightforwardly constructed using the steady-state generated by a semieternally inflating model, and extends to infinite negative cosmic time. Yet the arguments of [3, 4] (discussed in more detail in Sec. III) imply that it should be geodesically incomplete. This issue can be addressed with reference to the conformal diagram of the model, shown in Fig. 1 The background inflating spacetime is represented by the lightly shaded region. Each equal-time surface is intersected by an infinite number of bubbles (indicated by light-cones opening toward $\mathcal{J}^{+}$), which are concentrated along $\mathcal{J}^{+}$and near $i^{0}$.

The model thus far constructed (defined in the shaded region henceforth called "region I") is geodesically incomplete: all null geodesics (such as "Y" in Fig. 1), and all timelike geodesics (such as "X") other than the comoving ones, have only a finite proper time (or affine parameter) between a point in region I and coordinate time $t \rightarrow-\infty$ (see, e.g., [5]). Most geodesics thus "leave the spacetime" to their past, encountering $\mathcal{J}^{-}$, the limit surface of the flat equal-time surfaces as $t \rightarrow-\infty$. (On the conformal diagram this surface looks like a null cone emanating from a point at the bottom edge.)

Although the spacetime is geodesically incomplete 
there is no curvature blowup or other obvious pathology at $\mathcal{J}^{-}$, so the spacetime is extendible rather than singular. One may take the position that this sort of incompleteness is allowed, since the edge is outside of the future of any point in the region, and any given thing in the spacetime was made at some particular coordinate time $t>-\infty^{2}$. From this point of view, there is no clear reason to reject the model as defined in region I.

It seems quite reasonable, however, to ask instead how the manifold could be extended, and what could be in the extension. We start by extending the manifold to include $\mathcal{J}^{-}$, which is the boundary of the open set comprising region I. We shall see, as follows, that on $\mathcal{J}^{-}$the field must everywhere be in the false vacuum. Define $\phi(\lambda)$ as the field value at affine parameter $\lambda$ of a non-comoving geodesic starting at some arbitrary point $x_{i}$ in region I, where $\lambda$ increases away from $\mathcal{J}^{-}$. We know that for some affine parameter $\lambda_{\mathcal{J}}$, the geodesic encounters $\mathcal{J}^{-}$, and also that if our point is within a bubble, there is also a finite value $\lambda_{f}>\lambda_{\mathcal{J}}$ at which the geodesic leaves the bubble and enters the false vacuum $\phi_{f}$. Then for $\lambda<\lambda_{f}$, we have

$$
\lim _{\lambda \rightarrow \lambda_{\mathcal{J}}} \phi(\lambda)=\phi_{f}
$$

If we then require that the field be continuous along any geodesic, we then find that the field must be in the false vacuum $\phi_{f}$ everywhere on the surface $\mathcal{J}^{-}$. That is, at the semi-classical level of description, $\mathcal{J}^{-}$must be an infinite null surface of pure false vacuum, through which no bubbles pass.

Let us now examine the global classical structure of the background spacetime by momentarily neglecting semiclassical processes such as bubble nucleations. Then the manifold comprised of region I and $\mathcal{J}^{-}$is locally dS (constant Ricci scalar $R$ and vanishing Weyl curvature tensor) everywhere, with $\phi$ in the false vacuum $\left(\phi=\phi_{f}\right)$. This manifold can still be extended past $\mathcal{J}^{-}$, and the obvious extension is to complete dS spacetime. This is certainly $a$ solution compatible with our state at $\mathcal{J}^{-}$, and (as we will argue in Sec. IV and the Appendix) it seems likely to be unique. Thus we will take the maximal extension of the background spacetime to be full $\mathrm{dS}$ spacetime. That

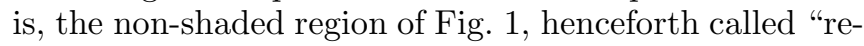
gion II" must simply be the rest of dS spacetime. Consider now a classical field in the background spacetime obeying a homogeneous hyperbolic equation. Given any point $P$ in region $\mathrm{I}$, almost all inextendible non-spacelike curves through $P$ intersect $\mathcal{J}^{-}$. Therefore specifying the field values on $\mathcal{J}^{-}$effectively poses a "characteristic initial value problem" 15, 16] with a unique solution everywhere in region I (this is the analog of the Cauchy

\footnotetext{
2 This was the view taken by investigators of the "cyclic model" 14] which is geodesically incomplete in a very similar way, and may be the view taken by the adherents of the classic steady-state model.
}

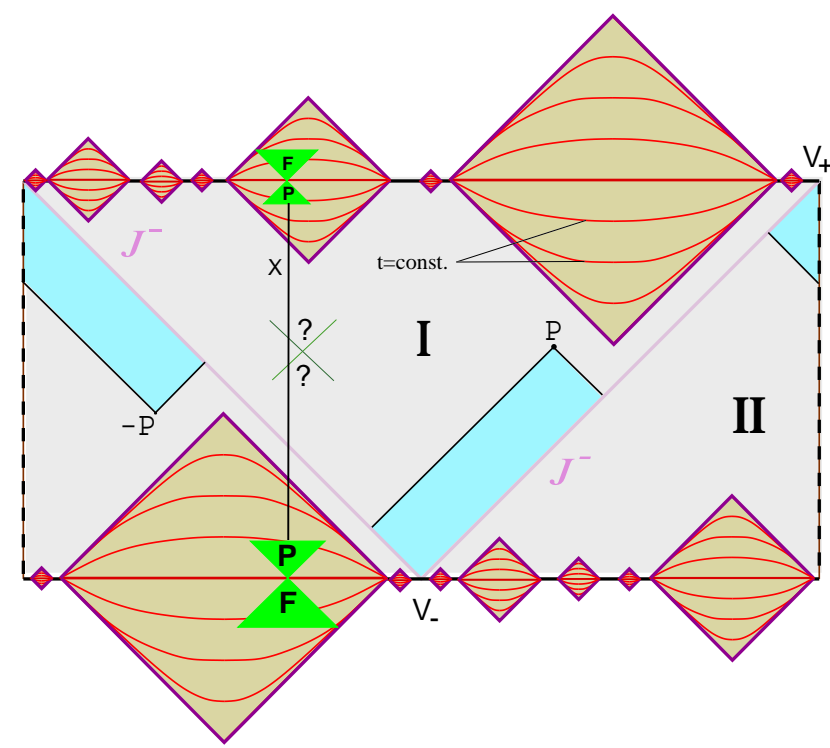

FIG. 2: Conformal diagram for eternal double-well inflation. Bubbles are open FRW regions; equal time slices shown as curved horizontal lines. For clarity we have not included bubble intersections. Also shown are past light-cones, cut off at $\mathcal{J}^{-}$, of both a point $P$ and its antipode $-P$ (note that $P$ and $-P$ are also reflected across the suppressed two-spheres in the $4 \mathrm{D}$ case).

problem, but with boundary conditions on a null surface; see Sec. IV and the Appendix for more details). Exactly the same argument can be made, however, for any point in region II. Thus specifying classical fields everywhere on $\mathcal{J}^{-}$determines their values everywhere in dS spacetime. This means that the conditions found to obtain on $\mathcal{J}^{-}$(by specifying the state in region I and requiring fields to be continuous) also determine the state in region II and we can extend our model to region II in an essentially unique way.

We may now examine the extension of the model to region II at the semi-classical level by including the bubble nucleations. The form of $U(\phi)$ indicates that bubbles must nucleate ${ }^{3}$ at a fixed rate per unit physical 4 -volume. In region $\mathrm{I}$, this led to an asymptotically steady-state bubble distribution which, when made exact, implied that there are no bubbles passing through $\mathcal{J}^{-}$. Thus in region II, though bubbles must nucleate at the required rate, none must pass through $\mathcal{J}^{-}$.

The only way this may occur is, in exact symmetry with region I, to have a steady-state bubble distribution on the flat slices of region II, with the bubbles opening away from $\mathcal{J}^{-}$. This is illustrated in Fig. 22, Region II is,

\footnotetext{
${ }^{3}$ Bubble nucleation has been perhaps most rigorously analyzed in Ref. [17], and the boundary conditions for bubble nucleation in our model correspond exactly to those analyzed in their study.
} 
then, a sort of mirror reflection of region I through $\mathcal{J}^{-}$. Thus the answer to the question of how the model can be extended, and what lies beyond $\mathcal{J}^{-}$, is that an essentially identical copy of region I lies in region II, connected by the infinite null surface $\mathcal{J}^{-}$.

This completes the basic specification of our model. The cosmology obeys the $\mathrm{CP}$ and PCP in that it admits a coordinatization such that all spatial slices are statistically homogeneous, and statistically the same as all others. There is no preferred time in this slicing, nor is there a cosmological singularity: the model is geodesically complete. Given the appropriate inflaton potential, any one of the bubbles could describe our observable surroundings.

\section{THE ARROW OF TIME}

If we consider all bubbles to expand with time, then Fig. 2 suggests that while in region I the future is toward the top of the diagram ("up"), in region II future lies toward the bottom ("down"). This leads us to the issue of the cosmological arrow of time (AOT): why does the time-asymmetric 2nd law of thermodynamics hold universally, given that fundamental physics is thought to admit a symmetry (CPT) that includes time-reversal? There is some consensus that if this question has an answer, it must ultimately be cosmological, with the time asymmetry resulting from some qualitative difference between cosmological "initial" and "final" conditions [18] that precludes a time-reversal (T-) symmetry of the physical state in any sub-region of the universe, and hence induces an AOT.

Although our our model has no "initial" conditions, it does have boundary conditions on $\mathcal{J}^{-}$(discussed in detail in Sec IV below) and we can discuss the AOT in light of them. To do so we must divide the universe into two types of sub-regions: those entirely outside of bubbles, and those partially or wholely within them. Outside of the bubbles (or alternatively near enough to $\mathcal{J}^{-}$) there is no local AOT: the description of such a region admits a time-reversal symmetry. Were we to hypothesize, for example, an imaginary observer outside of a bubble with its own AOT (pointing away from the time of its creation), that observer would see only T-invariant dS spacetime. The observer could not know whether it moved "up" or "down" on the conformal diagram, nor if it was in region I or II, nor if it crossed $\mathcal{J}^{-}$. What the observer is guaranteed, however, is that it will eventually be encountered by, and find itself within, a bubble.

The bubble interiors are not time-symmetric: within a bubble, there is a unique time direction in which the mean energy density decreases. This direction is away from the bubble wall/slow-roll inflation epoch, at which the FRW-region is known to be nearly homogeneous. If one bubble is to represent our observable surroundings, this direction must correspond to the time direction in which the entropy of an isolated system increases. It

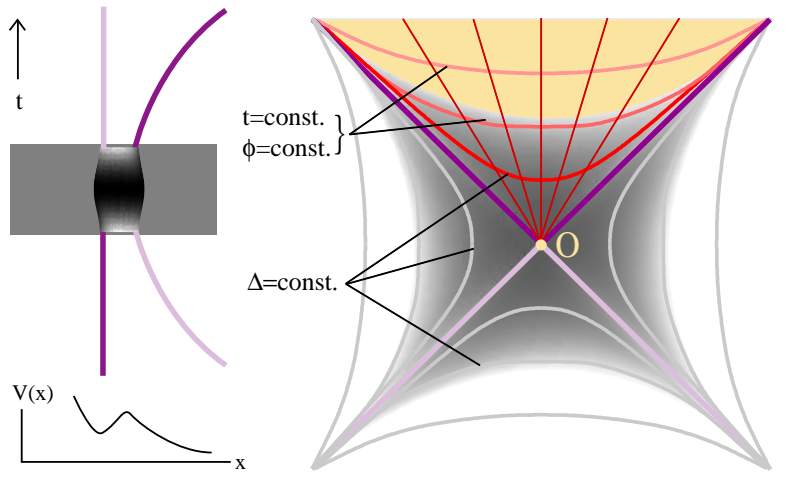

FIG. 3: The decay of: (left) an unstable particle, (right) an unstable vacuum state. In the particle case, the trajectory is classically describable to good precision at early and late times, but not near the decay (shaded region). Likewise, the inflaton $\phi$ is classically describable at large invariant distance $\Delta$ from the nucleation event at $O$, but not near it (shaded region). This quantum region connects the classically describable field configuration of the bubble interior to that of locally dS spacetime.

has been often argued, particularly by Penrose 19], that this connection arises because when gravity is included homogeneity corresponds to an extremely low-entropy state. We shall assume this correspondence here (and that the bubble does not begin in some very special state for which the density fluctuations decrease). Under this assumption the physical AOT within any bubble must point away from the bubble walls; globally this means that the AOT (where defined) points away from $\mathcal{J}^{-}$.

As illustrated in Fig. 22 one can therefore indeed draw timelike geodesics (such as "X") along which the physical AOT reverses, but the reversal always occurs to the past of any physical observer (all of which are within bubbles), and within a region (the locally dS spacetime) in which there is no well-defined physical AOT.

We have argued that within bubbles the physical laws, but not the physical state, admit a symmetry (CPT) including time-reversal, while outside of bubbles the laws and the state admit time symmetry; but what about the bubble nucleations themselves? Is there not some AOT telling them "which way" to nucleate, depending upon which side of $\mathcal{J}^{-}$they are on?

To clarify this point, consider the process of semiclassical bubble nucleation by analogy to the decay of an unstable particle, described by a potential $V(x)$ as in Fig. 3. Classically, one allowed trajectory is for the particle to sit at constant $x$ in the false minimum $x_{f}$ (vertical line on diagram); a second, equal-energy trajectory is for the particle to "bounce" off of the potential (curved line in diagram). A purely quantum description of the system (in the Schrödinger representation) would start, for example, with a Gaussian wave function centered at $x_{f}$ at some time $t=0$, and hence with the position expectation value $\langle\hat{x}\rangle \simeq x_{f}$. With time, the wave function 
spreads, and $\langle\hat{x}\rangle$ increases, eventually approaching the classical trajectory for $\langle\hat{x}\rangle \gg x_{f}$ (we could say roughly that the particle has "decayed" when $\langle\hat{x}\rangle$ changes significantly from $x_{f}$.) Note also that the same spreading would occur at $t<0$ so that this purely quantum description would be T-symmetric. Now, a semi-classical description of the system would describe the system classically at both early and late times, but with a quantummechanical transition connecting the classical trajectories at some given time. This transition time is random, and in an ensemble of such systems (as is required for a correct probabilistic description) would follow a probability distribution given by a WKB-type calculation of the decay rate. Near the transition time the system cannot be described classically; we must "shade out" the region where only a quantum description is accurate, as in Fig. 3

Bubble nucleation can be described semi-classically in a similar way. Here, we must attach allowed classical solutions of the field equations along some boundary that represents the nucleation event. To do this in a covariant way, this boundary must be a surface of zero proper distance, i.e. a null cone, as shown in Fig 3 A bubble nucleation "event" is thus comprised of a region (shown as the shaded upper quadrant) where a classical bubble interior solution applies, attached to locally dS regions by a "shaded out" region (within some proper separation squared of the nucleation point) where only a quantum description is valid. To produce a semi-classical description of a spacetime in which nucleation events occur, one must then populate it with these configurations in such a way that the nucleation sites are randomly situated and occur at the correct rate per unit four-volume, while the classical description, which applies far from the nucleation site, is in accord with the (classical) boundary conditions. When the classical boundary conditions are ours, given on $\mathcal{J}^{-}$, this yields the bubble distribution indicated in Fig. 2. The (semi-classical) boundary conditions do, then, control the time direction of bubble nucleation, not by introducing some locally-detectable AOT, but by controlling the allowed global configuration of bubble nucleation events.

There is one final "region" in which we can check the AOT: the entire universe. Interestingly, we here find that while each bubble nucleation event is non-timesymmetric, by virtue of the symmetry of the cosmological boundary conditions, the statistical description of the universe does admit a sort of T-symmetry. In Sec. V we will discuss the possibility of making this symmetry exact via an identification on the manifold. This raises the intriguing possibility of having a well-defined (and consistent among communicating observers) AOT for all observers even while the physical laws and the global physical description of the universe both admit a time-reversal symmetry.

\section{A. The singularity theorems}

Having examined the AOT, we may turn to the singularity theorems, for the AOT proves crucial in their analysis. Both the older theorems $[\underline{3}]$ and the newer theorem [4] assert that if certain conditions are satisfied everywhere within a spacetime, then not all past non-spacelike geodesics have infinite proper time or affine parameter. This indicates that the spacetime is either extendible or contains singularities.

The older theorem poses four such conditions, of which our model satisfies three, as does region I or II by itself. The fourth condition is that there exists a point $P$ such that there is a finite difference in volume between the interior of the PLC of $P$ and that of any point $P^{\prime}$ in the past of $P$. This is motivated by double-well inflation, and claimed as necessary for semi-eternal inflation (the argument being that if this condition does not hold, then any inflating point will find itself in a bubble at the next instant, with probability one). We argue that this condition is not quite necessary: what is required is that there be a finite volume in the region between the PLCs of $P$ and $P^{\prime}$ in which bubbles can nucleate toward $P$. Thus if, as in the proof of the theorem, the "past of $P$ " is taken to be the full volume interior to the light-cone pointing in the time direction away from which a bubble nucleated at $P$ would expand, then the fourth condition applies to region I alone (and correctly implies that it is extendible). But it would not apply to the full spacetime (which is neither extendible nor singular), because the relevant part of the light-cone extends only to $\mathcal{J}^{-}$.

The argument of the newer singularity theorem [4] consists of the definition of a local "Hubble parameter" $\mathcal{H}$ meant to represent the rate of divergence of neighboring comoving test particles, along with an argument that any region in which a suitable average $\mathcal{H}_{\text {av }}$ of $\mathcal{H}$ is greater than zero along all geodesics must be geodesically incomplete.

We understand Borde at al.'s argument as follows. One imagines some timelike or null test geodesic with affine parameter $\lambda$ in the spacetime in question, then attempts to construct a timelike vector field $u^{\mu}(\lambda)$ along the test geodesic into its past such that $\mathcal{H}_{\text {av }}$ (defined via $u^{\mu}$ ) exceeds zero. It is shown that this can only be achieved along some finite affine length of the test geodesic, since the imposed condition rapidly forces $u^{\mu}$ towards nullness. Borde et al. 4] then take their result to mean that an eternally inflating spacetime is past-geodesically incomplete.

We take the hypothetical satisfaction of their averaged Hubble parameter condition for all test geodesics as the implicit definition of what Borde et al. mean by an eternally inflating spacetime. The logic is that $u^{\mu}$ might be independent of test geodesic and simply be the velocity field of some set of comoving worldlines in the inflating spacetime. So what the theorem actually implies is that is it impossible to entirely cover a spacetime with such a set of worldlines in a way that allows all test geodesics 
cutting these worldlines to obey the Hubble parameter condition.

For illustration, let us consider dS space. We note that as pure $\mathrm{dS}$ is a maximally symmetric spacetime, it does not make sense to regard some parts as inflating and others not - one must break the symmetry by adding some other ingredients, and then frame a discussion in terms of them. Nevertheless, let us investigate if the geodesics defined by having fixed comoving coordinates themselves constitute a $u^{\mu}$ field satisfying $\mathcal{H}_{\text {av }}>0$. It turns out that $\mathcal{H}$ reduces to the usual $\dot{a} / a$ in this situation. In the closed slicing with metric

$$
d s^{2}=-d \tilde{t}^{2}+H^{-2} \cosh ^{2} H \tilde{t}\left(d \chi^{2}+\sin ^{2} \chi d \Omega_{2}^{2}\right),
$$

the coordinates cover all of $\mathrm{dS}$, so $u^{\mu}$ is globally defined. However, $\mathcal{H}<0$ for $\tilde{t} \leq 0$ so $\mathcal{H}_{\text {av }}$ goes negative there. Now consider using a single flat or open coordinate patch to define $u^{\mu}$. $\mathcal{H}_{\mathrm{av}}>0$ here (at least for the appropriate choice of time orientation). However, because neither of these coordinate patches covers the spacetime, neither does $u^{\mu}$. Furthermore, one may choose an infinite number of flat coordinatizations of $\mathrm{dS}$, each with a different null boundary where the $u^{\mu}$ construction fails. This makes it clear that the boundary of a given $u^{\mu}$ field cannot be unambiguously used to define an edge to an inflating region.

We do not believe that the global existence of a suitable $u^{\mu}$ is necessarily the best definition of what is meant by an eternally inflating spacetime. ${ }^{4}$ In particular, equating $\mathcal{H}$ with physical expansion entails a tacit assumption that the physical AOT is everywhere in accord with that defined by $u^{\mu}$. The model we have proposed could be covered by a congruence of geodesics (those comoving in two flat coordinate patches covering dS) that would yield $\mathcal{H}<0$ in some regions. However, we have argued that these regions may still be regarded as expanding with respect to the physical AOT defined by the cosmological boundary conditions.

In summary, both singularity theorems postulate conditions for a region to be "inflating", and find that such a region cannot be geodesically complete. However, interpreting these theorems as forbidding eternal inflation seem to us to require an unwarranted assumption about the global AOT independent of the cosmological boundary conditions.

\footnotetext{
4 Indeed the term "eternal inflation" has been used with a variety of meanings. For example, the recent paper [20] used it to describe models that are eternally inflating to the future, but simply geodesically complete to the past ( $\dot{a}$ and/or $\ddot{a}$ may go negative there). Such histories, with a globally-defined arrow of time, seem physically unrealistic with a typical mechanism for exiting inflation, since this would render them unstable to the formation of thermalized regions in the putatively eternal early phase 2].
}

\section{BOUNDARY CONDITIONS AND THE NULL BOUNDARY PROPOSAL}

We have seen how extending semi-eternal inflation to eternal inflation implies particular behavior on the infinite null surface $\mathcal{J}^{-}$. Here we discuss the converse, describing how the eternal double-well inflation model we have described can be specified by a particularly simple set of cosmological boundary conditions that are imposed on $\mathcal{J}^{-}$.

\section{A. On cosmological "initial" conditions}

The correct specification even of a complete set of physical laws does not by itself allow prediction of any physical system's behavior; these laws must be supplemented by boundary conditions that suffice to fully characterize the system being modeled. The Big-Bang ("BB") model essentially consists of a set of such boundary conditions for our observable cosmic surroundings: at some early time, our region was a hot, dense, nearly homogeneous and isotropic mixture of particles and fields in thermal and chemical equilibrium, in a nearly-flat expanding background geometry with scale-invariant gravitational potential perturbations of amplitude $10^{-5}$ on the scale of the cosmological horizon.

While these initial conditions yield predictions in excellent accord with astronomical observations, they are deemed by many to be too special: they seem to comprise an extremely small portion of some "ensemble of all possible initial conditions", using some (generally unspecified) measure (see e.g. 21]). The theory of inflation is widely accepted as a way to broaden the range of allow initial conditions by funneling a relatively wide class of physical conditions into satisfactory BB initial conditions 22].

This is perhaps reasonable as a stop-gap measure as long as the true (pre-inflation) initial conditions are unknown. But it does not solve the initial condition problem, for not all initial conditions will give rise to inflation [23], nor is it clear that all of those that do give inflation will yield a viable big-bang model [21]. Thus one must still assume that suitable conditions emerge from the initial singularity; whether this assumption corresponds to a "special" or "generic" condition seems illdefined without a description of the singularity, which would presumably require quantum gravity. But unless quantum gravity completely alters the way physical theories are applied, it is unlikely to yield a unique initial condition; it will still be necessary to supply a quantum state (or something similar). Even if it is possible to define the ensemble of all possible states, the question of how (and why) a state was "chosen" for our universe can only be a metaphysical one, and it seems that, just as in choosing physical laws, we can at best posit a state on some (possibly symmetrical or aesthetic or philosophical) grounds, derive its consequences, and compare them to 
the observed universe.

The same holds for a non-singular cosmological model such as that proposed in this paper, the basic structure of which is well-described by semi-classical physics, and where there can be no hiding of the cosmological boundary conditions in an initial singularity. We instead specify a particular set, and analyze them in terms of their simplicity, symmetry, and ability to correctly generate a big-bang like region that can describe our observable surroundings.

\section{B. (Semi-)classical boundary conditions for eternal inflation}

It is conventional to pose boundary conditions for a set of classical fields by specifying the fields (and generally their time derivatives) on a spacelike surface. This accords with the intuitive idea of specifying the state at an initial time. An alternative procedure, more relativistic in spirit because it does not assume a particular time coordinate, is to specify boundary conditions on a null surface such as the light-cone of a point. (See the Appendix and Refs. 15, 16, 24] for treatments of the null initial value problem.)

Our boundary surface $\mathcal{J}^{-}$is a null surface that, when drawn on the conformal diagram, looks like the light-cone of a point $V_{-}$at the bottom (i.e. "at" the conventional past timelike infinity) and the light-cone of a point $V_{+}$at the top; see Fig 21). As such, one might expect that one may determine fields (including the spacetime metric) throughout the space by specifying them on $\mathcal{J}^{-}$. The conformal mapping, however, hides the fact that $\mathcal{J}^{-}$is not a fully closed light-cone: there is always a nonzero physical volume (of order $\mathrm{H}^{-3}$ or greater) on any spacelike surface bounded by $\mathcal{J}^{-}$. We must therefore ask if specifying fields on $\mathcal{J}^{-}$suffices to fix them everywhere, or if there is information that may come "though the hole" at $V_{-}$and $V_{+}$. In the Appendix, we argue (using the Green function for a massive scalar field in dS) that the fields at $V_{ \pm}$are irrelevant (as long as they are reasonably well-behaved), as their effect is infinitely diluted.

We can thus pose boundary conditions for our cosmology at the classical level as:

1. There exists an infinite connected null hypersurface $\mathcal{J}^{-}$of topology $R \times S^{2}$, on which the 4-dimensional Weyl tensor vanishes and the $4 \mathrm{D}$ curvature scalar is constant.

2. The inflaton field, with a "double well" potential, is everywhere in the false vacuum on this surface.

3. On this surface, all classical fields are zero or are in minima of their potentials. This precludes any radiation propagating through $\mathcal{J}^{-}$.

This cosmology is classically very dull, as it is just de Sitter space everywhere with no dynamics. However, semi-classical bubble nucleations can, without affecting the fields on $\mathcal{J}^{-}$, create interesting dynamics by forming bubbles that open everywhere away from $\mathcal{J}^{-}$, and give rise to eternal inflation as described in Sec. II and shown in Fig. 2

\section{Quantum mechanical boundary conditions}

Although a fully quantum description of semi-classical phenomena - such as the nucleation of a bubble - is generally prohibitively complex, we may hope that, because our set of boundary conditions is so simple at a semi-classical level, it might be amenable to a simple quantum formulation, and it would be pleasing to specify our boundary conditions in an explicitly quantummechanical way. There are two ways one might think of doing this.

First, one might consider doing usual quantum field theory on de Sitter space, but with a particular choice of vacuum corresponding to our "false vacuum" classical conditions on $\mathcal{J}^{-}$. This might be done by putting the field in a Gaussian wavepacket around the false vacuum at (flat slicing) time $t$, as in [17], then taking a $t \rightarrow-\infty$ limit. One might then assume that this is sufficient to define the quantum state over all of de Sitter space. Alternatively, one could set such an initial state at the $\tilde{t}=0$ slice in global (closed) coordinates (7), i.e. on the slice through the "throat" of the dS hyperboloid. This surface could then be boosted (infinitely) to become $\mathcal{J}^{-}$. In either case, the procedure would be analogous to putting an unstable particle into a Gaussian in the false vacuum at $t=0$; away from $t=0$ in both time directions, the solution of the time-dependent Schrödinger equation would evolve away from the unstable "initial" state.

With our initial data surface being null, one might attempt to define the quantum state directly on it. This would be conceptually more pleasing, not requiring the limit through a sequence of spacelike surfaces as above. Further, a null surface formulation of initial data is rather elegant [16, 24]. However, unlike for a spacelike initial value surface, some points on $\mathcal{J}^{-}$are in causal contact. It is thus less easy to see how to move from a spacelike to a null boundary value surface in QFT, because fields do not commute at lightlike separated points. A similar effect occurs in so-called "light-cone quantization" approaches to QFT in Minkowski space [25], where one uses infinite null sheets (or lightfronts) instead of equal time slices, and fields again do not in general commute. The vacuum state for interacting fields is rather easier to define in the lightfront approach to QFT than in the usual spacelike approach to QFT, and one might expect this to hold in a proper null cone approach to QFT. Unfortunately, the authors know of no such formulation of QFT even in Minkowski space.

Thus while we suspect that our cosmological boundary conditions may correspond to a the specification of a rather simple quantum state, we leave this difficult problem for future work and here concentrate on 
a semi-classical description (though we return to QFT in Sec. $\nabla A$.

\section{Discussion of the null boundary proposal}

The boundary conditions we have proposed are extremely simple, in line with the view espoused above that one cannot avoid making a specific choice for cosmological boundary conditions, and that it is then reasonable to make a choice that is as simple and as highly symmetric as possible, rather than hope to choose a "generic" boundary condition.

\section{Relation to other proposals}

Our proposal is novel in that it requires no treatment of an initial singularity, and in that boundary conditions are placed on a null boundary that does not correspond to any particular cosmic "time". It does, however, have features in common with other proposals for specifying the state of the universe.

Penrose's Weyl Curvature Hypothesis (WCH) requires that initial singularities are constrained to have vanishing Weyl tensor, corresponding to a low-entropy state. This, he argues, gives rise to an arrow of time flowing away from the initial state. Our proposal is very similar, except of course that we impose the vanishing of the Weyl tensor on a lightlike slice running across the universe rather than at an initial singularity.

The Hartle-Hawking No Boundary Proposal (NBP) is a prescription for the wavefunction of the universe. It provides $\Psi\left(h_{i j}, \phi\right)$, the amplitude for a given three-metric and scalar field configuration on a spatial 3D surface. The construction is designed to suppress irregular configurations relative to more regular ones, and to thus favor simple and symmetric states. The notion of temporal evolution does not appear explicitly. However, one can associate histories with saddle-point approximations to the wavefunction, and it turns out that such universes are often smooth when they are young and small. Thus the NBP may be taken to imply that, at the semi-classical level, there should exist a spatial surface in a cosmological spacetime on which the boundary conditions are particularly simple. ${ }^{5}$. Our proposal rather uses a null surface (but see Sec.VIB below). It would be very interesting to develop a quantum prescription in a similar vein to the NBP which naturally leads to simplicity on certain null

\footnotetext{
${ }^{5}$ In the context of models with open-inflationary potentials, the NBP seems to favor histories in which the scalar field is everywhere in its true vacuum [26]. The NBP may still be relevant for inflation with the use of an anthropic constraint, or in a "top-down" approach to calculating quantum probabilities [27]. Starting off in the true vacuum is however not a problem for recycling models of inflation, as discussed below.
}

surfaces. For further details on both the WCH and NBP see 28 .

The tunneling approach to quantum cosmology [29] argues that the semi-classical universe emerges via a quantum tunneling event. In the context of models with open-inflationary potentials the proposal suggests that the universe, when first semi-classically describable, is most likely to be small and regular, with the field away from the true vacuum; future-eternal inflation can then ensue. The tunneling is supposed to have occurred out of a quantum gravitational chaos so severe as to preclude any space-time description. While our proposal also leads to inflation from semi-classical boundary conditions, it explicitly avoids any such extreme quantum gravitational regime.

Over the years Sakharov has discussed various cosmological models involving time-reversal (and CPT) invariance [30, 31, 32, 33. In these models, the universe is generally symmetric across a singular FRW bounce at $t=0$, at which universe assumes an especially simple state; away from this bounce entropy increases in both directions of time [31]. In [30] he hypothesizes that phenomena at $t<0$ are the CPT reflections of the phenomena at $t>0$. In [31, 32] he considers the possibility of an infinite chain of further bounces or cycles away from $t=0$ in both time directions, and also the possibility that the minimum-entropy surface might be one of maximum expansion rather than a singular one 31, 32. In a paper primarily about signature change [33] he alludes to possibility of non-singular time-reversal around a surface of minimal radius in a false vacuum state. Our proposal clearly has parallels with these ideas. We, however, concentrate on an infinite non-singular null surface, rather than a singular spacelike one. Moreover, with the concepts of semi-classical bubble nucleation and open inflation, we are able to provide a relatively complete physical picture.

\section{The horizon problem}

Inflation was originally conceived as a remedy for troubling issues concerning cosmological initial conditions [34], thus it is useful to compare how such shortcomings of the HBB model 22] are dealt with in our model. We focus on what is (aside from the singularity problem) perhaps the most vexing of these $\mathrm{HBB}$ difficulties: the horizon problem.

The horizon problem is generally framed as follows: choose two spatially antipodal points on the lastscattering surface. They are similar in temperature, yet their PLCs never intersect in a HBB cosmology, so there can be no causal connection between them. Inflation is generally thought to solve this problem, because with sufficient inflation, the PLCs will intersect. But this does not suffice for the points to have the same temperature, because the temperature at each point depends on data across its full PLC, and there is a portion of each PLC 
that does not intersect the other. Taking this into account, for the two points to have similar properties it must be assumed that there is sufficient inflation, and additionally that at inflation's beginning at time $t_{\text {inf }}$, the region to the past of the two points is homogeneous on length scales of order $H_{\mathrm{inf}}^{-1}$. But suppose there is a earlier epoch between some time $t$ and $t_{\text {inf }}$. Then at $t$ the patch must be homogeneous over a physical length scale of at least

$$
r(t) \sim \frac{a(t) H(t)}{a\left(t_{\mathrm{inf}}\right) H\left(t_{\mathrm{inf}}\right)} H^{-1}(t),
$$

where $H(t) \equiv \dot{a} / a$. If $a \propto t^{\alpha} \quad(\alpha=1 / 2$ for radiationdominated expansion) prior to $t_{\text {inf }}$, then $r /\left(H^{-1}\right) \simeq$ $\left[a / a\left(t_{\mathrm{inf}}\right)\right]^{(\alpha-1)}$. Thus it is necessary to postulate that our region was, at some initial time $t_{0}$, homogeneous over $\left[a\left(t_{\text {inf }}\right) / a\left(t_{0}\right)\right]^{(3-3 \alpha)}$ Hubble volumes. The horizon problem therefore persists if $t_{\text {inf }}>t_{0}$ and $\alpha<1$. There are two escapes available within inflation. The first is to set $t_{\text {inf }}=t_{0}=t_{\mathrm{pl}}$, so that the expansion is inflationary all the way back to the Planck time, before which one cannot speak of the expansion at all. The horizon problem is then greatly ameliorated, as it must only be assumed that some regions "emerging" from the Planck epoch are homogeneous over a relatively small (but $>1$; see [23]) number of Hubble volumes when they are first classically describable; but whether this constitutes a true solution will be seen only if and when the Planck epoch itself is understood. A second potential escape is to set $\alpha \geq 1$, or (more generally) for $\dot{a}$ to be non-decreasing, i.e. to have past-eternal inflation.

How, then, does the horizon problem look in the context of our eternal model with no initial time? Let us pose the problem in a slightly more general manner: when specifying the cosmological boundary conditions, must one do so over a region that is very large compared to some relevant physical volume such as $H^{-3}$ ? Posed this way, it might seem that because $\mathcal{J}^{-}$is an infinite surface, a strong horizon problem exists. ${ }^{6}$ This, however, is not necessarily the case. Imagine $\mathcal{J}^{-}$as the limit of a sequence of spacelike slices obtained by boosting the spacelike surface given by $\tilde{t}=0$ in global coordinates. Because each such surface has volume $2 \pi^{2} H^{-3}$, we might also attribute to $\mathcal{J}^{-}$the same finite invariant volume. ${ }^{7}$ Thus our construction would seem to ameliorate the horizon

\footnotetext{
6 This is apparently the case, for example, in the cyclic model 14], where if the cyclic behavior is to continue indefinitely into the future and past, it seems necessary to place cosmological boundary confitions on an infinite spacelike surface. This specification accords the same properties to points that are arbitrarily distant, and causally disconnected.

7 One can also consider other ways of taking a limit to $\mathcal{J}^{-}$, but this one seems most in accord with the symmetries of dS. Another approach to the problem is to rather consider some distance measure between any two points on $\mathcal{J}^{-}$. A dS-invariant quantity, in units where $H=1$, between points $P_{1}$ and $P_{2}$ in the embedding space (see Eq. (9) below) is given by $D\left(P_{1}, P_{2}\right)=$
}

problem (posed in terms of Hubble volumes within the boundary condition surface) to approximately the same degree as does inflation beginning in the Planck epoch, and much better than does inflation with an early quasiFRW phase.

\section{THE ELLIPTIC VIEW}

The model we have proposed consists of two indistinguishable regions, each comprising an eternally inflating universe with an AOT (where defined) pointing away from an infinite null surface which connects the two regions. The statistical identity of these universes, along with lack of a global physical time orientation, suggests some form of an old idea concerning dS, called "the elliptic interpretation" 8 that would identify the two universes.

The idea consists of deeming an event to be represented not by a single point of a spacetime manifold, but by a pair of antipodal points (defined below). This corresponds to a topological identification that, applied to our model, identifies regions I and II, and maps $\mathcal{J}^{-}$onto itself (the $R \times S^{2}$ manifold $\mathcal{J}^{-}$becomes $\left.\left(R \times S^{2}\right) / Z_{2}\right)$. This identification has been subject of some previous [36, 37, 38, 39] and recent [40] investigations.

Pure dS can be represented as a 4D hyperboloid

$$
-v^{2}+w^{2}+x^{2}+y^{2}+z^{2}=H^{-2}
$$

embedded in 4+1 D Minkowski space with metric

$$
d s^{2}=-d v^{2}+d w^{2}+d x^{2}+d y^{2}+d z^{2} .
$$

The elliptic interpretation consists of identifying each point $P$ with coordinates $(v, w, x, y, z)$ with its antipode $-P$ at $(-v,-w,-x,-y,-z)$. In the conformal diagram, this means that points such as $P$ and $-P$ of Fig. 2 are physically identified; the antipodal map $\mathcal{A}$ looks like a vertical reflection and a horizontal shift through one half of the diagram's horizontal extent (Note that although this map makes an orbifold of the embedding space, there

\footnotetext{
$-v_{1} v_{2}+w_{1} w_{2}+x_{1} x_{2}+y_{1} y_{2}+z_{1} z_{2}$ 11]. In general $-\infty<D<\infty$, whereas for two points on $\mathcal{J}^{-},|D| \leq 1$; they may thus be considered "close". The relation between $D$ and geodesic distance is, unfortunately, not always defined. The points are timelike or null separated, respectively, for $D>1$ or $D=1$. For $|D|<1$ points are connectable by a spacelike geodesic of length $\cos ^{-1}(D)<\pi$; but for $D \leq-1$ there is $n o$ geodesic connecting $P_{1}$ and $P_{2}$, although one does connect $P_{1}$ to the antipode $\bar{P}_{2}$ of $P_{2}$ because $D\left(P_{1}, \bar{P}_{2}\right)=-D\left(P_{1}, P_{2}\right)$. Under the identification of antipodal points (see below), any $P_{1}$ and $P_{2}$ are null-separated or spacelikeseparated by a geodesic distance $<\pi / 2 H$.

8 The etymology of this term is obscure to the authors. The elliptic view applied to spatial sections of $\mathrm{dS}$ was discussed first by de Sitter, who preferred it to the (now) conventional "spherical" view; and cited a letter from Einstein voicing the same preference [35]. Antipodally identifying in space and time was discussed in fond detail by Schrödinger 36.
} 


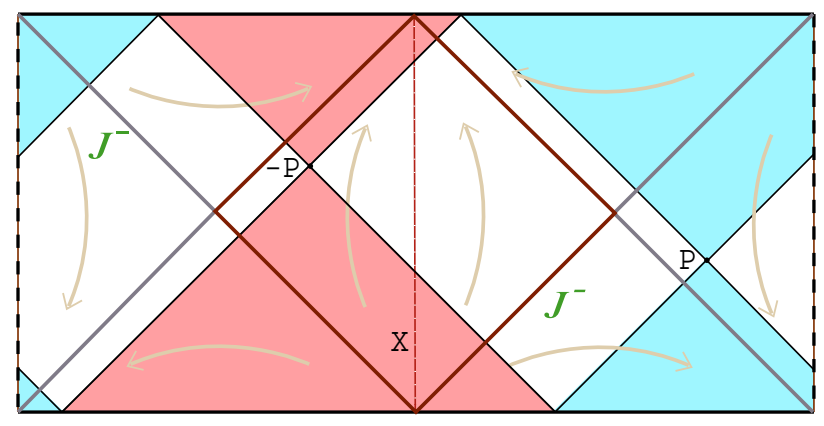

FIG. 4: Conformal diagram of dS including Killing vectors (arrows), a point $P$ and its antipode $-P$, along with their light-cones. The causal diamond of an observer following a geodesic "X" (comoving in the global coordinatization (7)) is the interior of the dark diamond, the antipodal copy of which is the light diamond.

are no fixed points in the $\mathrm{dS}$ hyperboloid, leaving the identified space a manifold).

At the classical level, the identification can be enforced by demanding that all fields in $\mathrm{dS}$ are symmetric or antisymmetric under $\mathcal{A}$, and that all sources have an accompanying antipodal copy. Parikh et al. 40] have argued that charge conjugation should be added to $\mathcal{A}$, and that the combination represents CPT. Indeed, in classical field theories at least, $\mathcal{A}$-symmetry automatically entails opposite charges at antipodal points. Consider, for example, a complex scalar field, satisfying $\phi(-P)=\phi(P)$. Then the global time derivative of the field at the antipode $-P$ is minus that at $P$, and hence the charge density $(q / 2 i) \sqrt{-g}\left(\phi^{*} \partial^{0} \phi-\phi \partial^{0} \phi^{*}\right)$ is opposite. However, in $3+1 \mathrm{D}$ at least, we do not recover the $\mathrm{PT}$ part of their argument. A particle at $P$ with 3 -momentum vector $\mathbf{p}=\left(p_{x}, p_{y}, p_{z}\right)$ has an antipodal copy at $-P$. By an argument like that of Parikh et al., parallel transporting the copy's trajectory back to $P$ takes the momentum to $\left(-p_{x}, p_{y}, p_{z}\right)$. This looks like parity followed by a rotation of $\pi$ about the $x$-axis. The same procedure, however, takes a small displacement $\mathbf{x}=(x, y, z)$ to $(x,-y,-z)$. The difference in sign arises because the 3 -momentum suffers an "automatic" time reversal under $\mathcal{A}$, whereas the 3 -displacement does not. Thus the orbital angular momentum $\mathbf{l}=\mathbf{x} \times \mathbf{p}$ transforms from $\left(l_{x}, l_{y}, l_{z}\right)$ to $\left(-l_{x}, l_{y}, l_{z}\right)$, just as the 3 -momentum does. But under parity $\mathrm{P}, \mathbf{x} \rightarrow-\mathbf{x}$ and $\mathbf{p} \rightarrow-\mathbf{p}$, while under $\mathrm{T}, \mathbf{x} \rightarrow \mathbf{x}$ and $\mathbf{p} \rightarrow-\mathbf{p}$. Therefore under PT (along with possible rotations), $\mathbf{p}$ and $\mathbf{l}$ transform oppositely. Only under $\mathrm{T}$ (with rotations) alone do they transform likewise. So unfortunately we cannot concur with the beguiling idea that CPT conjugate events occur at antipodally conjugate points in an elliptic universe. Rather, we must settle for a sort of CT conjugation between processes at antipodal points.

A second interesting (and favorable) feature of the an- tipodal identification, which holds in pure elliptic de Sitter space (edS), is as follows. An immortal observer in dS can only eventually "see" (i.e. be connected to via a non-spacelike geodesic emanating into the past of the observer) half of the space, the rest being hidden behind the observer's dS event horizon. Under the antipodal identification, however, this "hidden" space is exactly the same as the "visible" space. Likewise, the space behind the observer's particle horizon (the space not reachable by non-spacelike geodesics emanating from an observer toward its future) is the same as the space within it. In this precise sense, edS has no horizons. The notion that in edS each observer has "full information" about the space has been a prime motivation for the study of the space [36, 40].

Along with these appealing attributes however, and like our cosmological model, edS has some unconventional temporal features that may or may not be desirable. Because $\mathcal{A}$ includes time reversal, the spacetime is non-time-orientable: one cannot continuously divide nonspacelike vectors into two classes which can be labeled "future" and "past". Now, one may take the view 36 that since physics is essentially time-reversible, this poses no fundamental problem. Non-time-orientability does, however, have implications for quantum mechanics (see Sec. $\overline{V A}$ and [38, 39]). In addition, while physics may be time symmetric, our physical world manifestly is not, and this must be confronted in a cosmological model.

The identification of points near "past" infinity with those near "future" infinity also raises the specter of closed timelike curves (CTCs) and their accompanying paradoxes. The identification does not allow any selfintersecting timelike curves in perfect $\mathrm{dS}$ because the full light-cones of $P$ and $-P$ never intersect (the spacetime obeys the strong causality principle of [41]). For the same reason, no observer can see both an event and its antipodal copy. Note, though, that perturbations of dS that tend to make the conformal diagram "taller" 42] would allow timelike curves from a point to traverse the dS hyperboloid to the point's antipode.

These temporal features may not be what we are accustomed to, but perhaps we should not be surprised that any cosmology based on globally dS spacetime has an "unconventional" AOT. It is well known, for example, that while a patch of $\mathrm{dS}$ admits a static coordinatization, it admits no global timelike Killing vector; as shown in Fig. 4 the Killing vector is timelike only in half of the diagram, and moreover points toward the top of the diagram only in half of the region in which it is timelike, reversing completely between antipodal points. (In fact, the Killing vector field maps onto itself under $\mathcal{A}$ ).

Our model has much the behavior one would want in a cosmology based on edS. The physical AOT is defined only inside the bubbles, which may intersect (and hence compare time orientations) only within region I or region II. The two regions are separated by locally dS spacetime, where physics is fully time symmetric. Under the antipodal identification, the regions are equated, and the 
physical AOT is consistent everywhere that it can be compared by two physical observers. Only an imaginary observer that could travel "back in time" to leave a bubble, pass through $\mathcal{J}^{-}$, and encounter another bubble, could see that its own AOT agreed only with that of one of the two bubbles. (In fact, it would seem that in a nontime-orientable manifold the physical AOT must either be undefined in some regions, or must suffer a reversal along some surface. Thus a construction something like ours may well be necessary in any cosmology based on edS.)

However, an antipodally identified version of our model does not quite share all of the desirable properties of edS. The bubbles, with a larger curvature radius than the embedding space, allow the connection of antipodal (and hence identified) points by a timelike curve. These self-intersecting timelike curves (SITCs) are not however CTCs of the usual grandfather paradox sort. To follow such a SITC an imaginary observer would, for part of its journey, have to travel backward in (bubble) time. Moreover, when the two branches of the SITC meet, they have opposite time orientation as defined by an affine parameter along the curve. One might avoid these SITCs if the bubbles have a smaller curvature radius than the background space (as discussed below in Sec. VI). In this case, however, horizons would return, because there would be regions outside of an observer's horizon that are not identified with any region within the horizon.

While the elliptic interpretation is complicated by the presence of bubbles, the background space of our model is pure dS, and does benefit from the elliptic interpretation; all points on $\mathcal{J}^{-}$, for example, would be connectable by geodesics and have a maximal spacelike geodesic separation $\pi / 2 H$, and the volume of the boundary condition surface would be halved.

The elliptic interpretation was suggested by a symmetry in the statistical description of the bubbles, and it is interesting to ponder the connection between this statistical symmetry and $\mathcal{A}$. Classically, we may have an ensemble of systems each of which is not $\mathcal{A}$-symmetric, even while the statistical properties of the ensemble are. Without bubble nucleation, our universe $(\mathrm{dS})$ is $\mathcal{A}$-symmetric. The boundary conditions which determine the bubble distribution are $\mathcal{A}$-symmetric, therefore it seems necessarily true that the statistics of the bubble distribution are $\mathcal{A}$-symmetric. Given quantum mechanics, however, it is not clear how to relate a single member to the statistical properties (given by the wavefunction) without bringing in measurement theory, and we will leave the question aside for future consideration.

\section{A. QFT in edS}

The non-time-orientability of edS also makes quantum field theory on the space rather more subtle than in usual $\mathrm{dS}$. We hope to treat this subject in some detail in a forthcoming paper (see also [38, 39, 40]). Here we sketch a brief summary.

Consider a massive free scalar field $\phi(x)$ obeying some wave equation, for which we would like to construct a QFT in edS by defining a Hilbert space of states (including a vacuum state $|0\rangle)$, and the two-point function $\langle 0|\phi(x) \phi(y)| 0\rangle$. The latter can be decomposed into a commutator $D(x, y)$ and an anti-commutator (or "Hadamard") function $G^{(1)}(x, y)$. Under the antipodal identification, we would expect both $D$ and $G^{(1)}$ to be symmetric in some sense under the exchange of $x$ and/or $y$ with its antipode. How, then, might we define such a QFT? There are a number of ways, none of which seem entirely satisfactory.

First, one might just pick a particular "antipodally symmetric" vacuum state of full dS. Indeed, taking the $\alpha \rightarrow \infty$ limit of the " $\alpha$-vacua" appropriate for dS 43 does yield an $\mathcal{A}$-symmetric $G^{(1)}$. However, this does not have the usual short distance behavior of the Minkowski 2-point function. In addition, the commutator $D$ is independent of the state chosen, and has no antipodal symmetry. (One might also hope to find an $\mathcal{A}$-symmetric non-vacuum state with the correct short distance behavior, but this would still have the wrong commutator.)

A second approach would be to try to build an antipodally symmetric vacuum in a full dS background, by choosing a (global) time coordinate and decomposing the fields into global positive-frequency modes that are antipodally symmetric. The Fock vacuum would then as usual be the state destroyed by all annihilation operators. The problem with this approach is that any antipodally symmetric mode turns out to have vanishing Klein-Gordon norm when integrated over a Cauchy surface for all of dS [38], and the Fock construction breaks down.

A third approach employed in the literature 38, 39, 40] is to abandon the hope of a Fock vacuum, and to simply enforce the antipodal symmetry at the level of the Green functions, by writing antipodally symmetrized versions of the fields, and computing the resulting twopoint functions in terms of the two-point functions of unidentified dS. One problem with this approach is that it becomes somewhat unclear why the anticommutator should take this value, as the construction seems to lack an underlying quantum-mechanical motivation. Another problem is that while the anticommutator so obtained is antipodally symmetric, the same procedure yields a commutator that vanishes identically.

A fourth approach is to define the vacuum in terms of a mode decomposition over only part of $\mathrm{dS}$ (such as a "causal diamond" 11]), where the modes can be consistently positive or negative frequency (so that a Fock representation exists), then provide a prescription for defining correlators between any two points in the space in terms of correlators within this region. This approach turns out to be promising; for one or both points in the causal diamond or its antipodal copy, and for a particular choice of state (a mixed thermal one at twice the usual de Sitter Hawking temperature), both $D$ and $G^{(1)}$ can be 
made to have the correct symmetry. The problem arises when both points are outside of the causal diamond and its copy; in this case the commutator turns out to vanish for timelike separated points, and not for spacelike separated points. It is unclear whether this makes sense.

In short, defining a satisfactory QFT in edS is rather difficult; the difficulties stem primarily from the commutator function, because it is not symmetric under timereflection, while the anti-commutator is. It is then difficult for both functions to be symmetric under $\mathcal{A}$, which includes time-reflection. It is possible [40] that string theory in edS will make more sense than in $\mathrm{dS}$. It is also conceivable that the elliptic view could emerge from a correct quantum treatment of $\mathrm{dS}$, and that the described troubles stem from doing QFT on a fixed background not included in the dynamics. This would be an interesting issue to pursue in string theory or other theories of quantum gravity. For now we must leave it there, and return to eternal inflation.

\section{GENERALIZATIONS AND EXTENSIONS}

We have constructed our eternally inflating universe using an "open inflation" double-well potential and demanding that the inflaton $\phi$ rest in the false vacuum everywhere on the infinite null surface $\mathcal{J}^{-}$. But these choices are not unique, and the general principles of our construction can be extended to models employing different potentials, or different boundary condition surfaces.

\section{A. Different inflaton behaviors}

A simple change in our model can be induced by leaving $V(\phi)$ fixed, but demanding that $\phi$ rest in the true, rather than false, vacuum on $\mathcal{J}^{-}$. Although the field is now in a (positive) stable vacuum, bubbles of false vacuum may still be able to nucleate [44]. Assuming this indeed occurs, the effect of each bubble can be enclosed within a light-cone, and the distribution of these lightcones is essentially the same as for bubbles of true vacuum, so all of the arguments of Sec I go through. Now, within these bubbles of false vacuum new bubbles of true vacuum can form, one of which could describe our observable universe. At late (bubble) times each bubble interior would approach dS, dominated by the true vacuum (presumably of the magnitude we currently appear to observe), in which false vacuum bubbles may nucleate (and so on, ad-infinitum). This is a realization of the "recycling universe" of [5]. Because bubbles are nested infinitely deep, the structure of this universe appears exceedingly complicated. But the global structure at the outermost level of bubbles is known (and is as in Fig. 2), because we have specified it using explicit cosmological boundary conditions. One might argue that this scenario would have yet simpler boundary conditions than the scenario in which the inflaton is placed in the false vacuum on $\mathcal{J}^{-}$. Since the lowest vacuum state must be positive for this scenario to work, it also has the potential to connect with the presently observed positive vacuum energy.

A somewhat similar scenario can be realized using "chaotic inflation" potentials such as $V(\phi)=\lambda \phi^{4}+V_{0}$. Here we set the inflaton to rest at $\phi=0$ on $\mathcal{J}^{-}$, and require $V_{0}>0$ so that the space is locally dS near $\mathcal{J}^{-}$. This is, again, a stable configuration, yet regions of large potential may "nucleate" in this dS background as highly improbable quantum fluctuations in the field. If such a region nucleates at high enough $\phi$, it would provide the potential seed for the sort of eternal inflation envisioned by Ref. [46], in which quantum fluctuations in $\phi$ dominate over classical rolling so that inflation become eternal. While the structure of the resulting region becomes extremely complicated, it can again be contained within a light-cone of some point in the original (dS) embedding space, so that the global structure of the universe (filled with such light-cones) is still understandable, and again looks like Fig. 2

\section{B. Different boundaries}

We were led to the null surface $\mathcal{J}^{-}$by constructing an eternal model with a time-translation symmetry. But the same sort of boundary conditions we apply on $\mathcal{J}^{-}$could also be applied to a spacelike initial surface, such as the $\tilde{t}=0$ surface in the global coordinatization (7) of dS. Such boundary conditions might be closely tied in to the Hartle-Hawking NBP, as discussed above. The universe now has a preferred (and initial) time, and constitutes "semi-eternal" inflation in both the $\tilde{t}<0$ and $\tilde{t}>0$ regions. The same arguments concerning the AOT apply here: it is undefined near $\tilde{t}=0$ (outside all bubbles), and defined in bubbles, pointing away from $\tilde{t}=0$. If the initial surface maps onto itself under the antipodal map, we can apply the antipodal identification to the universe. This model bears a stronger resemblance to the earlier ideas of Sakharov 30, 31, 32, 33] than does our proposal using the null $\mathcal{J}^{-}$.

Spacelike surfaces are easily deformable into other spacelike surfaces, whereas the same is not true for null surfaces (see e.g. [47]). Thus spacelike surfaces are in some sense less constrained than null ones and thus maybe less appropriate in any attempt, such as ours, to specify cosmological boundary conditions in the most economical way. In addition, the resulting universe would not obey the Perfect Cosmological Principle, having a preferred time at $t=0$. As discussed above, we also might conjecture that the quantum state corresponding to null boundary conditions is simpler. In general, however, there seems to be no strong argument against such a boundary condition surface as compared to a null surface. 


\section{SUMMARY AND CONCLUSIONS}

We have investigated the possibility of making "futureeternal" inflation eternal also to the past. Starting with a de Sitter spacetime background dominated by the falsevacuum energy in which true-vacuum bubbles can form, we specify that the bubble distribution at any time is in exactly the steady-state configuration asymptotically approached by semi-eternal inflation. All bubbles are then equivalent, and the statistical distribution of bubbles admits both a time-translation and space-translation invariance in the background inflating space.

The described region ("region I") has no initial time, but has a null boundary $\mathcal{J}^{-}$that is the limiting surface as $t \rightarrow-\infty$ of the flat spatial sections. The steadystate configuration at $t>-\infty$ corresponds to the inflaton field being in the false vacuum state everywhere on $\mathcal{J}^{-}$, with no incoming radiation from $\mathcal{J}^{-}$. This surface can be reached by a past-directed geodesic of finite proper length from any point in the space, so region I is geodesically incomplete; this is the "singularity" pointed to by theorems purporting that past-eternal inflation is impossible 3, 4]. But the space can (and should) be extended, as the state on $\mathcal{J}^{-}$also constitutes boundary conditions for the region past $\mathcal{J}^{-}$if the manifold is extended. These boundary conditions imply that a duplicate copy of region I exists on the other side of $\mathcal{J}^{-}$. Together region I and the new "region II" constitute a geodesically complete (i.e. non-singular) inflating cosmology that admits a coordinatization in which the background space and bubble distribution are time-translation and spacetranslation invariant.

The null surface $\mathcal{J}^{-}$constitutes a cosmological boundary condition surface on which the universe is classically in a particularly simple and symmetric state. Although $\mathcal{J}^{-}$is an infinite null surface, some of its points are in causal contact, and one might attribute a finite invariant volume of $O\left(H^{-3}\right)$ to it. Therefore specifying boundary conditions on it is not like specifying them on an infinite spacelike hypersurface (which would lead to a severe "horizon problem").

It might be possible to construct a quantum state corresponding to our classical boundary conditions on $\mathcal{J}^{-}$by taking a null-limit of spacelike sections on which the wave functional describing the fields is centered on the desired classical state. But an explicitly null quantum formulation of our null boundary proposal has not been provided and would constitute an interesting future study.

It is widely thought that the "arrow of time" is connected with cosmological boundary conditions. In our model, time flows away from $\mathcal{J}^{-}$, and the AOT is consistent among all physical observers that can compare it. The AOT is not, however, defined globally, and in our model the statistical description of the universe admits a global symmetry that includes time-reversal.

This symmetry, along with the presence of two duplicate, non-communicating universes, motivates - though does not require - formally identifying antipodal points on the manifold. We, and others, have studied this identification on de Sitter space classically and quantummechanically. Antipodally identified (or "elliptic") dS has the virtues that it is causally stable and observers have no event horizons. It has the disadvantage that its non-time-orientability makes defining a reasonable quantum field theory difficult. With antipodal identification our model is more economical as the two duplicate universes are identified; however not all of the attractive features of "pure" edS remain.

Our model can be generalized to other inflaton potentials (such as for chaotic inflation), and therefore allows one to partially understand the global structure of the eternally inflating spaces that result. One may also use an analogous construction to specify a semi-eternally inflating but non-singular universe by placing boundary conditions on a spacelike section.

Our primary conclusion is that it is possible, using only "standard" ingredients underlying popular models for inflation, to specify simple cosmological boundary conditions on an infinite null surface that lead to past- and future-eternal inflation. Such a universe would obey the same Perfect Cosmological Principle that governs a semieternally inflating universe long after its beginning. If our construction survives scrutiny, and can be specified at the fully quantum (or quantum-gravitational) level within a theory of fundamental physics, it could serve as the basis for a realistic cosmology that avoids a cosmological singularity, a beginning of time, or a creation of the universe "from nothing".

\section{Acknowledgments}

We thank Alex Vilenkin for helpful comments on a draft of this paper. AA is supported by a grant from the W.M. Keck foundation. SG is supported in part by US Department of Energy grant DE-FG02-91ER40671.

\section{APPENDIX}

Here we review Green functions and the initial value problem in some detail, focusing for simplicity on the inhomogeneous scalar wave equation in fixed background spacetimes. We are interested in how much information must be specified, and where, in order to fix the field throughout spacetime.

\section{Defining Green functions}

Imagine that our scalar field $\phi$ satisfies

$$
\left(\square-m^{2}\right) \phi=q\left(x^{\mu}\right),
$$

where $q$ is an arbitrary source term, and we wish to determine $\phi$ at some point $P$ with coordinates $x_{P}$. This is possible given $\phi$ and its time derivative on some complete spacelike slice through the past light-cone (PLC) of $P$. Let us see why this is so, and whether this is the only suitable set of initial data for the problem. 


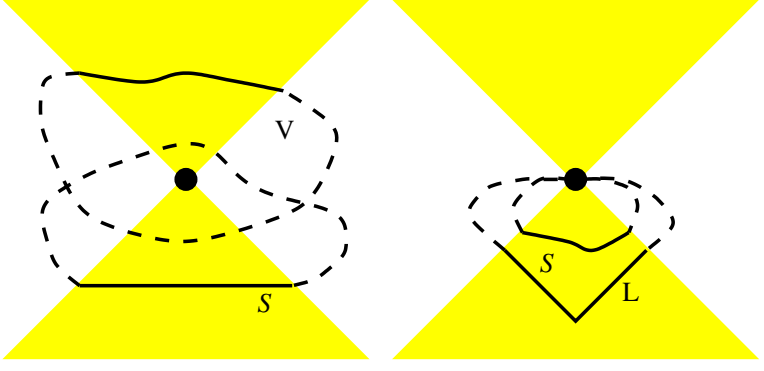

FIG. 5: Possible choices of $\mathcal{V}$ for different Green functions. The solid lines indicate the regions $\mathcal{S}$ of $\partial \mathcal{V}$ which contribute to the integral. On the left, we have volumes suitable for the retarded and advanced Green functions $G_{R}$ and $G_{A}$, and on the right we have volumes suitable for the commutator function $G_{C}$.

First introduce another function $G$, which depends on $P$ and is assumed to satisfy

$$
\left(\square-m^{2}\right) G=s\left(x^{\mu}\right),
$$

where $s$ is to be chosen. Taking $G$ times (11) minus $\phi$ times (12) and integrating through some four dimensional volume $\mathcal{V}$ yields

$$
\begin{array}{r}
\int d^{4} x \partial_{\mu}\left(G \sqrt{-g} g^{\mu \nu} \partial_{\nu} \phi-\phi \sqrt{-g} g^{\mu \nu} \partial_{\nu} G\right) \\
=\int \sqrt{-g}(q G-s \phi) d^{4} x
\end{array}
$$

This allows determination of $\phi(P)$ in terms of its values elsewhere by a suitable choice of $s, G$, and $\mathcal{V}$.

To effect this, we must isolate $\phi(P)$, either on the left or right hand side of (13). Let us first use the RHS. Take $s$ to be a $\delta$-function at $P$ (or, more carefully, a function peaked near $P$ that can be taken to a $\delta$-function limit). Then choosing $\mathcal{V}$ to enclose $P$ gives $\phi(P)$ for the second term on the RHS of Eq. (13). Using Gauss's law, this equation can now be used to express $\phi(P)$ as a surface integral over of $\phi$ and its derivative over the boundary $\partial \mathcal{V}$ of $\mathcal{V}$, plus a volume integral over $\mathcal{V}$ of the source alone.

To fix $G(x)$, we must choose boundary conditions specific to our choice of $s$. Two conventional choices are to require $G$ to vanish either to the future or to the past of $P$. In the first case $G$ is known as the "retarded" Green function and may be denoted $G_{R}$; in the second case it is "advanced" $\left(G_{A}\right)$.

We must now choose $\mathcal{V}$ (see Fig. 5). It turns out (see, e.g., [15]) that $G_{A} \neq 0$ only on and within the FLC of $P$, and that $G_{R} \neq 0$ only on and within the PLC of $P$. Hence only a segment $\mathcal{S}$ of $\partial \mathcal{V}$ contributes to Eq. (13), enabling us to deduce $\phi(P)$ using only data on some connected surface making a complete span through either the
FLC or the PLC of $P$. The usual choice, consistent with our standard ideas about causality, is to pick a spacelike surface of constant time (see left side of Fig. 5). Then evaluation of Eq. (13) requires $\phi$ and $\dot{\phi}$ on $\mathcal{S}$. For a more general $\partial V$, we require $\phi$ and its normal derivative on $\mathcal{S}$. (Note that this surface need not be everywhere spacelike, but if not then the data must be self-consistent.) The case of present interest is that of a null surface, for which the normal lies everywhere within the surface itself (this is made possible by the Lorentzian signature of the spacetime.) Then specifying $\phi$ on the surface also specifies the normal derivative of $\phi$ on it. A suitable null surface is, for example, the forward light-cone $L$ of a point $Q$ to the past of $P$; now we need only know $\phi$ on the surface $\mathcal{S}$ where this FLC of $Q$ is within the PLC of $P$ (see right side of Fig. 5). (If the data near $Q$ is suitably regular, the non-smooth nature of the surface at $Q$ is unimportant.) In the same spirit, one might consider a "wedge" such as the boundary of the future of the segment of a line in the past of $P$.

Let us now consider the second way of isolating $\phi(P)$, using the LHS of Eq. (13). Set $s=0$, and choose some piece of $\partial \mathcal{V}$ to be spacelike and run through $P$. Then choose $G$ to vanish everywhere on this surface. Further, choose it to have a $\delta$-function at $P$ in its normal derivative; the integral then picks up a contribution proportional to $\phi(P)$. This choice comprises boundary conditions for $G$, and the function they determine, which we denote $G_{C}$, now vanishes outside the (full) light-cone of $P$ and satisfies the homogeneous version of Eq. (12). Choosing now the remainder of $\partial V$ to close off to the future or to the past of $P$, we again may determine $\phi(P)$ once data is given on a complete slice $\mathcal{S}$ through one of the lightcones. $G_{C}$ may be called the commutator function, because it turns out to be equal to $-i$ times the commutator of a free quantum field (at least in a globally hyperbolic spacetime). Note also that $G_{C}=G_{A}-G_{R}$ : subtracting the (inhomogeneous) equations governing $G_{R}$ and $G_{A}$ leaves a homogeneous equation for the difference. Then the difference must vanish outside the light-cone of $P$, just as for $G_{C}$.

In all cases, $G$ depends on the point $P$, and so may be considered as a function of two variables, $x$ and $x_{P}$. Because $\phi$ satisfies (11), it turns out that the $G$ also satisfies (11) with respect to $x_{P}$, at least away from $x_{P}=$ $x$.

\section{Green functions for Minkowski and dS spacetimes}

Let us now outline a procedure to obtain some Green functions for massive scalar fields in certain spacetimes, using a slightly unconventional but perhaps more intuitive method not relying on the usual Fourier techniques or $i \epsilon$-prescriptions. We start with the commutator function for a massless field in 3+1 D Minkowski space,

$$
G_{C}^{M_{0}}=\operatorname{sgn}(t) \delta\left(t^{2}-r^{2}\right) / 2 \pi,
$$


with coordinates such that $P$ is at the origin. That this solves $\square \phi=0$ can be seen by expanding out the $\delta$-function and comparing to a general superposition of incoming and outgoing spherically symmetric waves. (A nice discussion of this function is found in [48].) Note that this is only non-zero on the light-cone itself, vanishing even inside the cone. This is a special property of massless fields in even-dimensional spacetimes (see [15] for more details.)

To obtain the solution for the massive field in Minkowski space, we start by considering

$$
G_{1} \equiv f\left(t^{2}-r^{2}\right) \operatorname{sgn}(t) \Theta\left(t^{2}-r^{2}\right) \text {. }
$$

The sgn and $\Theta$ functions enforce the gross features of the behaviour that we require. Writing $\square$ using spherical polar coordinates, we find

$$
\begin{aligned}
\left(\square-m^{2}\right) G_{1} & =\operatorname{sgn}(t) \Theta\left(t^{2}-r^{2}\right)\left(\square-m^{2}\right) f \\
& -4 f(0) \operatorname{sgn}(t) \delta\left(t^{2}-r^{2}\right)
\end{aligned}
$$

Let us choose $f\left(\tau^{2}\right)$ (where here $\tau^{2}=t^{2}-r^{2}$ ) such that $\left(\square-m^{2}\right) f=0$ inside the light-cones; a general solution is $f\left(\tau^{2}\right)=A J_{1}(m \tau) / \tau+B Y_{1}(m \tau) / \tau$ where $J_{1}$ and $Y_{1}$ are Bessel functions as in Ref. [49]. The first term of Eq. [15] is then zero. For the second term, we notice that it is basically the solution to the massless problem that we've already found. So let us simply add the massless solution $G_{C}^{M_{0}}$ from Eqn. (14) to our ansatz $G_{1}$. Since $\square G_{C}^{M_{0}}=0$, we have left $-m^{2} G_{C}^{M_{0}}$ on the RHS. By choosing $A=$ $-m / 4 \pi$ and $B=0$, we obtain a complete cancellation, leaving us with our desired solution. Thus the full Green function is

$$
G_{C}^{M}=\frac{\operatorname{sgn}(t)}{2 \pi}\left(\delta\left(\tau^{2}\right)-\frac{m \Theta\left(\tau^{2}\right) J_{1}(m \tau)}{2 \tau}\right) .
$$

We may now consider generalizing Eq. (16) to other spacetimes such as dS. First, let us note that we took $f$ above to be a function of the proper time $\tau$ from the origin, within the light-cone. Thus the commutator function was invariant under Lorentz boosts. This suggests that in $\mathrm{dS}$, for example, we make our commutator inside the forward light-cone invariant under isometries which leave $P$ fixed. This is the same as saying that it should be a function of the proper time from the origin alone. One can define this quantity in terms of an "angle" in the 5D embedding space [11] (see footnote to Sec IVD 2), but perhaps a more intuitive way to proceed is as follows. A patch of $\mathrm{dS}$ can be covered by coordinates in which the metric is the same as that for an open expanding FRW universe, with scale factor $H^{-1} \sinh H t$. The proper time along the geodesics representing comoving observers is just given by the coordinate time $t$. These geodesics all intersect at a point as $t \rightarrow 0$, which we choose to be $P$, and they cover all of the interior of the FLC of $P$. We therefore need only find an appropriate spatially homogeneous solution of the massive wave equation in the coordinate patch of the open slicing of $\mathrm{dS}$. The equation reads:

$$
\frac{1}{\sinh ^{3} H t} \partial_{t}\left(\sinh ^{3} H t \partial_{t} \phi\right)+m^{2} \phi=0
$$

To solve this [50], write $\phi=H \chi / \sinh H t$ and set $z=$ cosh $H t$ to obtain

$$
\left(1-z^{2}\right) \chi_{, z z}-2 z \chi_{, z}+\left(2-\frac{m^{2}}{H^{2}}-\frac{1}{1-z^{2}}\right) \chi=0
$$

which is Legendre's equation [49] with $\nu=-1 / 2+$ $\sqrt{9 / 4-m^{2} / H^{2}}$ and $\mu=-1$. The solution to this equation which is regular as $z \rightarrow 1$ is $P_{\nu}^{\mu}(z)$. In terms of $t$, this tends to $H t / 2$ as $t \rightarrow 0$ independent of $m^{2}$, hence $\phi$ tends to $H / 2$.

We can now use this result to deduce the form of the commutator over all of dS space. Extend the meaning of $\tau^{2}$ to be the signed geodesic distance squared between $P$ and the point in question. Note that we do not consider the point to be in the light-cone of the antipode of $P$, since no geodesic exists which connects it to $P$. In this case we rather define $G_{C}$ to be zero (but see our discussion of the antipodal quantum commutator above.) Near $P$ the space is locally Minkowskian, so we can compare to our Minkowksi result to obtain:

$$
G_{C}^{d S}=\frac{\operatorname{sgn}(t)}{2 \pi}\left[\delta\left(\tau^{2}\right)-\frac{m^{2} \Theta\left(H^{2} \tau^{2}\right)}{2 \sinh H \tau} P_{\nu}^{-1}(\cosh H \tau)\right]
$$

Here by $t$ is meant some suitable generalization of Minkowski time, with $\operatorname{sgn}(t)$ thus serving to make $G_{C}^{d S}$ be of opposite sign in the future and past light-cones, and $\tau=+\sqrt{\tau^{2}}$. The $\operatorname{sgn}(t) \delta\left(\tau^{2}\right)$ should be interpreted as the generalization of the equivalent term in the Minkowski result.

\section{Domains of dependence}

Given our Green functions, we would like to investigate to what extent fixing the fields on a null-cone $L$ of a point $Q$ determines the field values within that cone. We are particularly interested in the importance of the field near $Q$, as our cosmological boundary surface $\mathcal{J}^{-}$can be considered the light-cone with $Q$ "at" past-timelike infinity.

Take, as a first example, the Green function $G_{R}$ for a massless field such as the scalar field in $4 \mathrm{D}$ Minkowski space with $G_{R}$ from Eq. (14). Because $G_{R}$ has support only on the PLC of $P$, the field at $P$ depends only on the intersection of $P$ 's PLC and $L$. This indicates two things. First, specifying data on $L$ explicitly determines the field everywhere inside $L$ (i.e. everywhere in the future of $Q$ ). Second, the field at any point $P$ inside $L$ does not depend on $\phi(Q)$; we might thus consider $Q$ to be irrelevant in terms of what occurs within $L$. This, however, hides 
a subtlety: while $G_{r}$ and $\phi$ on $L$ always allow the construction of a valid solution of the field equations, there is no guarantee that the field so obtained is continuous with the field specified on $L$ unless some assumptions of field regularity at the vertex $(Q)$ are made: if we wish to evaluate $\phi$ on $L$ to check that we have a genuine solution to our boundary value problem, then we must either know $\phi(Q)$, or assume that the field is regular as $Q$ is approached along $L$, so that we can extend the field to $Q$ by continuity.

For a massive field the strict "Huygen's Principle" does not hold; while much of the contribution to the integral in Eq.(13) comes from the PLC of $P$ where $G_{R}$ is singular (see 15] for some discussion of this "generalized Huygen's principle"), there is also a contribution from inside $P$ 's PLC because $G_{R}$ is everywhere nonzero there.

In the dS case, $G_{R}$ as given by Eq. (19) falls off exponentially as $\tau \rightarrow \infty$; thus we may expect that when $L$ is taken to be $\mathcal{J}^{-}$, where the vertex lies "at" $\tau=\infty$, the field values inside $\mathcal{J}^{-}$will not depend on the field at the vertex (where by "at the vertex" we mean within $\mathcal{J}^{-}$in the limit $\tau \rightarrow \infty)$. More explicitly, we may consider a boundary surface comprised of $\mathcal{J}^{-}$at $\tilde{t}>\tilde{t}_{0}$ for some $\tilde{t}_{0}$, closed by the spacelike surface $\tilde{t}=\tilde{t}_{0}$, where $\tilde{t}$ and $\tilde{t}_{0}$ are in the global closed coordinates. Then the field integral (13) consists of an integral along $\mathcal{J}^{-}$, and an integral over the $\tilde{t}=\tilde{t}_{0}$ hypersurface, which has a finite physical volume of order $H^{-3}$. But then as $\tilde{t}_{0} \rightarrow-\infty$, $\tau \rightarrow \infty$, so this second contribution will vanish unless either the average of the field or its $\tilde{t}$-derivative blow up faster than $G_{R}^{-1}$. Hence we expect that the field at any point in the space will depend only on the values on $\mathcal{J}^{-}$, and not on the field behavior at past infinity, as long as the fields are assumed to be suitably finite and regular.

This argument might also be applicable to gravity; the equations governing the Weyl conformal tensor can be cast, at the linear level, as wave-equations for a spin-2 field, and boundary conditions can be specified on a null surface such as $\mathcal{J}^{-}$[24]. We therefore expect that our boundary conditions on $\mathcal{J}^{-}$determine the Weyl tensor uniquely, and therefore determine the spacetime to be pure dS when bubble nucleations are neglected.
[1] A. Linde, D. Linde and A. Mezhlumian, Phys. Rev. D 54, 2504 (1996); A. Vilenkin, Phys. Rev. D 46, 2355 (1992).

[2] See, for example, A.H. Guth, Phys. Rept. 333, 555 (2000) and references therein; A.D. Linde, Phys. Lett. B175, 395 (1986); A.D. Linde in The Very Early Universe, edited by G.W. Gibbons, S.W. Hawking and S.T.C. Siklos (Cambridge University Press, Cambridge, England, 1983); P.J. Steinhardt, same volume.

[3] For a review, see A. Borde and A. Vilenkin, Int. J. Mod. Phys. D5 (1996) 813-824.

[4] A. Borde, A.H. Guth and A. Vilenkin, gr-qc/0110012

[5] A. Aguirre and S. Gratton, Phys. Rev. D 65 (2002) 083507.

[6] A.H. Guth, Phys. Rev. D 23, 347 (1981).

[7] M. Bucher, A.S. Goldhaber and N. Turok, Phys. Rev. D $\mathbf{5 2}, 3314$ (1995).

[8] J.R. Gott, Nature (London) 295, 304 (1982); K. Yamamoto, M. Sasaki, and T. Tanaka, Astrophys. J. 455, 412 (1995); A. Linde and A. Mezhlumian, Phys. Rev. D 52, 6789 (1995)

[9] A. Vilenkin, Phys. Rev. D 46, 2355 (1992).

[10] A.H. Guth and E.J. Weinberg, Nucl. Phys. B212, 321 (1983).

[11] For reviews of de Sitter space, see: Ref. 41]; M. Spradlin, A. Strominger, and A. Volovich, hep-th/0110007

[12] S. Coleman and F. De Luccia, Phys. Rev. D 21, 3305 (1980).

[13] B. Mandelbrot, The Fractal Geometry of Nature, (W.H. Freeman and Co., New York, 1983).

[14] P.J. Steinhardt and N. Turok, hep-th/0111030

[15] R. Courant, Methods of Mathematical Physics: Vol. II, Partial Differential Equations (New York: Interscience Publishers, John Wiley \& Sons, 1962).

[16] R. Penrose, Gen. Rel. and Grav. 12, 225 (1980).

[17] V.A.Rubakov and S.M.Sibiryakov Theor. Math. Phys. 120, 1194 (1999).
[18] See, e.g., H.D. Zeh, "The physical basis of the direction of time", (Berlin: Springer-Verlag, 1999).

[19] R. Penrose, in "Quantum Gravity 2: A Second Oxford Symposium", ed. C.J. Isham, et al. (Oxford: Clarenden Press), 1981.

[20] G.F.R. Ellis and R. Maartens, qr-qc/0211082

[21] R. Penrose, in "Fourteenth Texas Symposium on Relativistic Astrophysics, Dallas, Tex., 1988" (New York: Annals of the New York Academy of Sciences, 1989); S. Hollands and R. Wald; gr-qc/0205058 N. Turok, Class. Quantum Grav. 19, 3449 (2002).

[22] A. Linde, "Particle physics and inflationary cosmology", (Switzerland: Harwood Acedemic Publishers, 1990).

[23] T. Vachaspati and M. Trodden, Phys. Rev. D 61, 023502 (2000).

[24] R. Penrose and W. Rindler, "Spinors and Space-Time, Vol. I", (Cambridge: Cambridge University Press, 1984).

[25] T. Heinzl, Lect. Notes Phys. 572, 55 (2001), hep-th/0008096

[26] S. Gratton and N. Turok, Phys. Rev. D 63, 123514 (2001).

[27] S.W. Hawking and T. Hertog, Phys. Rev. D 66, 123509 (2002).

[28] S.W. Hawking and R. Penrose, The Nature of Space and Time (Princeton University Press, Princeton, New Jersey, USA, 1996).

[29] A. Vilenkin, Phys. Rev. D 27, 2848 (1983).

[30] A. D. Sakharov, JETP Lett. 5, 24 (1967).

[31] A. D. Sakharov, Sov. Phys. JETP 52, 349 (1980).

[32] A. D. Sakharov, Sov. Phys. JETP 56, 705 (1982).

[33] A. D. Sakharov, Sov. Phys. JETP 60, 214 (1984).

[34] A. H. Guth, Phys. Rev. D 23, 347 (1981).

[35] W. de Sitter, M.N.R.A.S 78,3 (1917).

[36] E. Schrödinger, Expanding Universes (Cambridge University Press, Cambridge, England, 1956).

[37] H. Schmidt, Fortschr. Phys. 41, 179 (1993).

[38] G. Gibbons, Nuc. Phys. B271, 497 (1986). 
[39] N. Sanchez, Nuc. Phys. B294, 1111 (1987); J.L. Friedman and A. Higuchi, Phys. Rev. D 52, 5687 (1995).

[40] M. Parikh, I. Savonije and E. Verlinde, hep-th/0209120

[41] S.W. Hawking and G.F.R. Ellis, The large scale structure of space-time (Cambridge University Press, Cambridge, England, 1973).

[42] F. Leblond, D. Marolf and R. C. Myers, JHEP 0206, 052 (2002).

[43] E. Mottola, Phys. Rev. D 31, 754 (1985); B. Allen, Phys. Rev. D 32, 3136 (1985).

[44] K. Lee and E.J. Weinberg, Phys. Rev. D 36, 1088 (1987).

[45] J. Garriga and A. Vilenkin 1998, Phys. Rev. D 57, 2230
(1998).

[46] A. Linde, D. Linde and A. Mezhlumian, Phys. Rev. D 49, 1783 (1994).

[47] R. Bousso, Rev. Mod. Phys. 74, 825 (2002).

[48] P.A.M. Dirac, Lectures on Quantum Field Theory, (New York: Belfer Graduate School of Science), 1996.

[49] M. Abramowitz and I. Stegun, "Handbook of Mathematical Functions" (New York: Dover Publications, 1972).

[50] M. Sasaki, T. Tanaka and K. Yamamoto, Phys. Rev. D 51, 2979 (1995). 